\title{
A limit of the quantum Rényi divergence
}

\author{
Nilanjana Datta and Felix Leditzky \\ Statistical Laboratory, University of Cambridge, \\ Cambridge CB3 0WB, United Kingdom
}

September 23, 2013

\begin{abstract}
Recently, an interesting quantity called the quantum Rényi divergence (or "sandwiched" Rényi relative entropy) was defined for pairs of positive semi-definite operators $\rho$ and $\sigma$. It depends on a parameter $\alpha$ and acts as a parent quantity for other relative entropies which have important operational significances in quantum information theory: the quantum relative entropy and the min- and max-relative entropies. There is, however, another relative entropy, called the 0-relative Rényi entropy, which plays a key role in the analysis of various quantum informationprocessing tasks in the one-shot setting. We prove that the 0-relative Rényi entropy is obtainable from the quantum Rényi divergence only if $\rho$ and $\sigma$ have equal supports. This, along with existing results in the literature, suggests that it suffices to consider two essential parent quantities from which operationally relevant entropic quantities can be derived - the quantum Rényi divergence with parameter $\alpha \geq 1 / 2$, and the $\alpha$-relative Rényi entropy with $\alpha \in[0,1)$.
\end{abstract}

\section{Introduction}

A fundamental quantity in quantum mechanics which plays a particularly relevant role in quantum information theory is the $\alpha$-relative Rényi entropy (see e.g. [1] and [2]), where the parameter $\alpha \in[0, \infty) \backslash\{1\}$. Its applications range over problems as diverse as binary state discrimination, state compression, information transmission, catalytic transformations of states, as well as finding bounds on the communication complexity of certain distributed computation problems (see e.g. [3] and references therein).

Recently, a generalization of this quantity was proposed independently by Wilde et al. 44 and Müller-Lennert et al.[5]. The resulting quantity, which was referred to as "sandwiched" Rényi relative entropy in the former paper, and quantum Rényi divergence in the latter 1 , is defined as follows (see also [6, 7]): For a density matrix $\rho$, a positive semi-definite operator $\sigma$ and a parameter $\alpha \in(0,1) \cup(1, \infty)$,

$$
D_{\alpha}(\rho \| \sigma):=\frac{1}{\alpha-1} \log \left[\operatorname{Tr}\left(\sigma^{\frac{1-\alpha}{2 \alpha}} \rho \sigma^{\frac{1-\alpha}{2 \alpha}}\right)^{\alpha}\right]
$$

For $\alpha \geq 1$, we set $D_{\alpha}(\rho \| \sigma)=\infty$ if $\operatorname{supp} \rho \nsubseteq \operatorname{supp} \sigma$. Here supp $\rho$ denotes the support of $\rho$, i.e., the span of eigenvectors of $\rho$ corresponding to non-zero eigenvalues. The above definition

\footnotetext{
${ }^{1}$ In this paper, we use the name quantum Rényi divergence
} 
is easily extended to the case in which $\rho \geq 0$ but $\operatorname{Tr} \rho \neq 1$, and our result (Theorem 1 below) is valid for it too.

For certain ranges of values of the parameter $\alpha$, the quantum Rényi divergence, $D_{\alpha}(\rho \| \sigma)$, has been proved to possess a host of interesting properties desirable for a divergence measure (see e.g. [4, 5, 6, 7]). In particular, for $\alpha \geq 1 / 2$ it satisfies the data-processing inequality [6, 7], i.e., it is monotonous under any completely positive trace-preserving (CPTP) map $\Lambda$ :

$$
D_{\alpha}(\Lambda(\rho) \| \Lambda(\sigma)) \leq D_{\alpha}(\rho \| \sigma) \text { for } \alpha \geq 1 / 2 .
$$

It satisfies joint convexity for $1 / 2 \leq \alpha \leq 1$ [6], and positivity if $\rho$ and $\sigma$ are both density matrices [4, 5, 7].

Moreover, the quantum Rényi divergence acts as a parent quantity for other relative entropy quantites. If $[\rho, \sigma]=0$, it reduces to the standard $\alpha$-relative Rényi entropy:

$$
\widetilde{D}_{\alpha}(\rho \| \sigma):=\frac{1}{\alpha-1} \log \left(\operatorname{Tr}\left(\rho^{\alpha} \sigma^{1-\alpha}\right)\right),
$$

and hence can be viewed as a generalization of $\widetilde{D}_{\alpha}(\rho \| \sigma)$ to the non-commutative case. In the limit $\alpha \rightarrow 1$, it reduces to the quantum relative entropy

$$
D(\rho \| \sigma):=\operatorname{Tr}(\rho \log \rho)-\operatorname{Tr}(\rho \log \sigma),
$$

whereas in the limit $\alpha \rightarrow \infty$ it reduces to the max-relative entropy [8]:

$$
D_{\max }(\rho \| \sigma):=\inf \left\{\gamma: \rho \leq 2^{\gamma} \sigma\right\} .
$$

Moreover, for $\alpha=1 / 2$ it is equal to the min-relative entropy [9]:

$$
D_{\min }(\rho \| \sigma):=-2 \log \|\sqrt{\rho} \sqrt{\sigma}\|_{1} .
$$

The min- and max- relative entropies are of particular relevance in the fast-developing field of one-shot quantum information theory. This is because characterizations of operational quantities (e.g. capacities of channels, data compression limits, entanglement cost, distillable entanglement etc.) in the one-shot setting can be obtained in terms of smoothed entropies [10] derived from them (see e.g. [11] and references therein). There is, however, another relative entropy which is of particular relevance in the one-shot setting. This is the 0-relative Rényi entropy

$$
\widetilde{D}_{0}(\rho \| \sigma):=\lim _{\alpha \rightarrow 0} \widetilde{D}_{\alpha}(\rho \| \sigma)=-\log \left(\operatorname{Tr} \Pi_{\rho} \sigma\right),
$$

where $\Pi_{\rho}$ denotes the projection onto the support of $\rho$ (and obviously satisfies $0 \leq \Pi_{\rho} \leq$ $\left.I, \operatorname{Tr}\left(\Pi_{\rho} \rho\right)=1\right)$. It has a simple operational interpretation in the problem of binary state discrimination; namely, for two density matrices $\rho$ and $\sigma, 2^{-\widetilde{D}_{0}(\rho \| \sigma)}$ is equal to the minimum probability of type II error, under the condition that the type I error is strictly zerd 2 . If instead one allows the probability of type I error to be bounded above by $\varepsilon>0$, then the minimum probability of type II error is given by a similar expression, but with $\widetilde{D}_{0}(\rho \| \sigma)$ replaced by a smoothed version obtained by replacing $\Pi_{\rho}$ in (2) by an operator $0 \leq A \leq I, \operatorname{Tr}(A \rho) \geq 1-\varepsilon$, and maximizing over all such operators. This yields the quantity

$$
\max _{\substack{0 \leq A \leq I \\ \operatorname{Tr}(\bar{A} \rho) \geq 1-\varepsilon}}(-\log (\operatorname{Tr}(A \sigma))) .
$$

\footnotetext{
${ }^{2}$ For a binary POVM $\{E, I-E\}$ used for the state discrimination, we define the probabilities of type I and type II errors respectively as $\alpha(\rho, \sigma)=\operatorname{Tr}((I-E) \rho)$ and $\beta(\rho, \sigma)=\operatorname{Tr}(E \sigma)$ respectively.
} 
The above quantity is usually referred to as the hypothesis testing relative entropy [12, 9], and denoted as $D_{H}^{\varepsilon}(\rho \| \sigma)$. It has various interesting properties and applications in one-shot quantum information theory, e.g. bounds on the one-shot capacity of a classical-quantum channel can be expressed in terms of it [12]; it also arises in the analysis of one-shot lossy data compression [14. An alternative smoothed version of $\widetilde{D}_{0}(\rho \| \sigma)$ occurs naturally in the characterization of the one-shot entanglement cost under local operations and classical communication (LOCC) [13. In fact, the (unsmoothed) 0-relative Rényi entropy provides an explicit characterization of the entanglement cost of a bipartite state in the case of perfect entanglement dilution in the one-shot setting [13]: the minimum number of Bell states needed to create a single copy of a bipartite state $\rho_{A B}$ perfectly, by two distant parties (Alice and Bob, say) using LOCC is given by the following expression:

$$
\min _{\mathcal{E}} \max _{\sigma_{R}}\left\{-\widetilde{D}_{0}\left(\rho_{A R}^{\mathcal{E}} \| I_{A} \otimes \sigma_{R}\right)\right\},
$$

where the maximization is over density matrices $\sigma_{R}$ on a Hilbert space $\mathcal{H}_{R}$, and the minimization is over all possible pure-state ensembles $\mathcal{E}=\left\{p_{i},\left|\psi_{A B}^{i}\right\rangle\right\}$ such that $\rho_{A B}=\sum_{i} p_{i}\left|\psi_{A B}^{i}\right\rangle\left\langle\psi_{A B}^{i}\right|$, and $\rho_{A R}^{\mathcal{E}}=\operatorname{Tr}_{B} \rho_{A B R}^{\mathcal{E}}$ where

$$
\rho_{A B R}^{\mathcal{E}}=\sum_{i} p_{i}\left|i_{R}\right\rangle\left\langle i_{R}|\otimes| \psi_{A B}^{i}\right\rangle\left\langle\psi_{A B}^{i}\right| .
$$

Hence, it is evident that the 0-relative Rényi entropy plays an important role in one-shot quantum information theory. Thus, it is interesting to ask whether the quantum Rényi divergence also acts as a parent quantity for it, in the general case in which $[\rho, \sigma] \neq 0$. In this paper, we answer this question in the affirmative when $\rho$ and $\sigma$ have equal supports. However, the 0-relative Rényi entropy is not obtainable from the quantum Rényi divergence when the supports of $\rho$ and $\sigma$ are unequal. We prove the latter via an explicit counterexample.

Our result is given by Theorem 1 below, and its implications are discussed in the Conclusions.

\section{The result}

Throughout the paper $\mathcal{H}$ denotes a finite-dimensional Hilbert space. We denote by $\mathcal{P}(\mathcal{H})$ the set of positive semi-definite operators on $\mathcal{H}$ and by $\mathcal{D}(\mathcal{H})$ the set of density operators on $\mathcal{H}$, i.e. operators $\rho \in \mathcal{P}(\mathcal{H})$ with $\operatorname{Tr} \rho=1$.

Our result is stated in the following theorem.

Theorem 1 For $\rho \in \mathcal{D}(\mathcal{H})$ and $\sigma \in \mathcal{P}(\mathcal{H})$ with $\operatorname{supp} \rho=\operatorname{supp} \sigma$, the following identity holds

$$
\lim _{\alpha \rightarrow 0} D_{\alpha}(\rho \| \sigma)=\widetilde{D}_{0}(\rho \| \sigma) .
$$

The above identity does not necessarily hold if $\operatorname{supp} \rho \subset \operatorname{supp} \sigma$.

The claim in (3) follows from Lemma 3 and Lemma 8 below. In Lemma 3 we prove that the left hand side of (3) is upper bounded by $\widetilde{D}_{0}(\rho \| \sigma)$, for any $\rho \in \mathcal{D}(\mathcal{H})$ and $\sigma \in \mathcal{P}(\mathcal{H})$ with $\operatorname{supp} \rho \subseteq \operatorname{supp} \sigma$. In Lemma 8 we prove that, if the supports of $\rho$ and $\sigma$ are identical, then the left hand side of (3) is also lower bounded by $\widetilde{D}_{0}(\rho \| \sigma)$.

Finally we provide an explicit, analytical counterexample to (3) for the case in which the supports of $\rho$ and $\sigma$ are not equal. 


\subsection{Upper bound for $\lim _{\alpha \rightarrow 0} D_{\alpha}(\rho \| \sigma)$}

Bounding the quantum Rényi divergence $D_{\alpha}(\rho \| \sigma)$ from above by the $\alpha$-relative Rényi entropy $\widetilde{D}_{\alpha}(\rho \| \sigma)$ is achieved by employing the Araki-Lieb-Thirring inequality [17, 18, which we state here:

Theorem 2 Let $A$ and $B$ be positive semi-definite operators on a finite-dimensional Hilbert space $\mathcal{H}$ and $q \geq 0$. Then the following holds:

(i) $\operatorname{Tr}\left(B^{1 / 2} A B^{1 / 2}\right)^{r q} \leq \operatorname{Tr}\left(B^{r / 2} A^{r} B^{r / 2}\right)^{q}$ for $r \geq 1$

(ii) $\operatorname{Tr}\left(B^{1 / 2} A B^{1 / 2}\right)^{r q} \geq \operatorname{Tr}\left(B^{r / 2} A^{r} B^{r / 2}\right)^{q}$ for $0 \leq r<1$

This allows us to prove the following

Lemma 3 For all $\alpha>0, \rho \in \mathcal{D}(\mathcal{H})$, and $\sigma \in \mathcal{P}(\mathcal{H})$, with $\operatorname{supp} \rho \subseteq \operatorname{supp} \sigma$, we have $D_{\alpha}(\rho \| \sigma) \leq \widetilde{D}_{\alpha}(\rho \| \sigma)$.

Proof. Let $\beta:=\frac{1-\alpha}{2 \alpha}$ and consider $\alpha \in(0,1)$. Setting $q=1, r=\alpha, A=\rho$ and $B^{1 / 2}=\sigma^{\beta}$ in Theorem 2 (ii) yields

$$
\operatorname{Tr}\left(\sigma^{\beta} \rho \sigma^{\beta}\right)^{\alpha} \geq \operatorname{Tr}\left(\sigma^{\frac{1-\alpha}{2}} \rho^{\alpha} \sigma^{\frac{1-\alpha}{2}}\right)=\operatorname{Tr}\left(\rho^{\alpha} \sigma^{1-\alpha}\right)
$$

where the equality sign follows from the cyclicity of the trace. Since the logarithm is a monotonous function and $\alpha-1<0$ for $\alpha \in(0,1)$, we obtain from (4) that

$$
D_{\alpha}(\rho \| \sigma)=\frac{1}{\alpha-1} \log \operatorname{Tr}\left(\sigma^{\beta} \rho \sigma^{\beta}\right)^{\alpha} \leq \frac{1}{\alpha-1} \log \operatorname{Tr}\left(\rho^{\alpha} \sigma^{1-\alpha}\right)=\widetilde{D}_{\alpha}(\rho \| \sigma),
$$

proving the claim for $\alpha \in(0,1)$. The claim for $\alpha>1$ follows analogously from Theorem 2 (i), as was mentioned in [4].

Remark 4 Note that Lemma 3 implies that

$$
\lim _{\alpha \rightarrow 0} D_{\alpha}(\rho \| \sigma) \leq \lim _{\alpha \rightarrow 0} \widetilde{D}_{\alpha}(\rho \| \sigma)=\widetilde{D}_{0}(\rho \| \sigma) .
$$

2.2 Lower bound for $\lim _{\alpha \rightarrow 0} D_{\alpha}(\rho \| \sigma)$ when $\operatorname{supp} \rho=\operatorname{supp} \sigma$

To obtain a lower bound of $\lim _{\alpha \rightarrow 0} D_{\alpha}(\rho \| \sigma)$, when $\operatorname{supp} \rho=\operatorname{supp} \sigma$, we use the concept of "pinching" defined as follows (see e.g. [19, Appendix A]):

Definition 5 (Pinching) Given an operator $B \in \mathcal{P}(\mathcal{H})$, let $B=\sum_{I=1}^{n(B)} b_{i} P_{i}$ be its spectral decomposition, where $n(B)$ is the number of distinct eigenvalues of $B$, and $P_{i}$ is the projection corresponding to the eigenvalue $b_{i}, i=1,2, \ldots, n(B)$. The following map

$$
\mathcal{E}_{B}: \omega \in \mathcal{P}(\mathcal{H}) \mapsto \mathcal{E}_{B}(\omega):=\sum_{i=1}^{n(B)} P_{i} \omega P_{i} \in \mathcal{P}(\mathcal{H})
$$

is called pinching. Note that the set of projectors $\left\{P_{i}\right\}_{i=1}^{n(B)}$ satisfy the completeness relation $\sum_{i=1}^{n(B)} P_{i}=I$, and are said to constitute a projection-valued measure (PVM).

With this definition, the following lemma holds: 
Lemma 6 Given a PVM $\left\{P_{i}\right\}_{i=1}^{n(B)}$, for all $\rho \in \mathcal{D}(\mathcal{H})$,

$$
\rho \leq n(B) \mathcal{E}_{B}(\rho) .
$$

Proof. See [19, Appendix B, Lemma 2].

We employ a slight adaption of the above lemma, given by the following corollary, which can easily be proved using Gram-Schmidt orthogonalization.

Corollary 7 Consider $\sigma \in \mathcal{P}(\mathcal{H})$ with $n:=\operatorname{dim} \mathcal{H}$ and its eigenvalue decomposition

$$
\sigma=\sum_{j=1}^{n} s_{j} \pi_{j}, \quad \text { with } \pi_{j}=\left|\psi_{j}\right\rangle\left\langle\psi_{j}\right|,
$$

with eigenvalues $s_{j}$ listed in decreasing order, repeated according to their multiplicities, and rank-one projectors $\pi_{j}=\left|\psi_{j}\right\rangle\left\langle\psi_{j}\right|$ taking care of degeneracies. Then for all $\rho \in \mathcal{D}(\mathcal{H})$ :

$$
\rho \leq n \sum_{j=1}^{n} \pi_{j} \rho \pi_{j} .
$$

Using the above corollary, we are able to prove that, if $\operatorname{supp} \rho=\operatorname{supp} \sigma$ a lower bound for $\lim _{\alpha \rightarrow 0} D_{\alpha}(\rho \| \sigma)$ is indeed given by $\widetilde{D}_{0}(\rho \| \sigma)$ :

Lemma 8 For $\rho \in \mathcal{D}(\mathcal{H})$ and $\sigma \in \mathcal{P}(\mathcal{H})$, with $\operatorname{supp} \rho=\operatorname{supp} \sigma$ we have

$$
\lim _{\alpha \rightarrow 0} D_{\alpha}(\rho \| \sigma) \geq \widetilde{D}_{0}(\rho \| \sigma)
$$

Proof. Let $\rho \in \mathcal{D}(\mathcal{H})$ and $\sigma \in \mathcal{P}(\mathcal{H})$ with $\operatorname{supp} \rho=\operatorname{supp} \sigma$. Let $n:=\operatorname{dim} \mathcal{H}$ and $\beta:=\frac{1-\alpha}{2 \alpha}$, with $\alpha \in(0,1)$. Furthermore, let $\sigma$ have the eigenvalue decomposition given by (5). Let the analogous eigenvalue decomposition for $\rho$ be $\rho=\sum_{i=1}^{n} r_{i}\left|\phi_{i}\right\rangle\left\langle\phi_{i}\right|$. We also define

$$
\mu_{j}:=\sum_{i=1}^{n} r_{i}\left|\left\langle\phi_{i} \mid \psi_{j}\right\rangle\right|^{2} .
$$

Then, by Corollary 7 ,

$$
\begin{aligned}
C_{\alpha}:=\sigma^{\beta} \rho \sigma^{\beta} & \leq n \sum_{j=1}^{n} \pi_{j}\left(\sigma^{\beta} \rho \sigma^{\beta}\right) \pi_{j} \\
& =n \sum_{j=1}^{n} s_{j}^{2 \beta} \pi_{j} \rho \pi_{j} \\
& =n \sum_{j=1}^{n}\left(s_{j}^{2 \beta} \sum_{i=1}^{n} r_{i}\left|\left\langle\phi_{i} \mid \psi_{j}\right\rangle\right|^{2}\right) \pi_{j} \\
& =n \sum_{j=1}^{n}\left(s_{j}^{2 \beta} \mu_{j}\right) \pi_{j} \\
& =n Q_{\alpha} .
\end{aligned}
$$

where $Q_{\alpha}:=\sum_{j=1}^{n}\left(s_{j}^{2 \beta} \mu_{j}\right) \pi_{j}$. From (하) and the operator monotonicity of $x \mapsto x^{\alpha}$ for $0<\alpha<1$, it follows that

$$
C_{\alpha}^{\alpha} \leq n^{\alpha} Q_{\alpha}^{\alpha}
$$


Since $Q_{\alpha}$ is already spectrally represented, we have that

$$
Q_{\alpha}^{\alpha}=\sum_{j=1}^{n} s_{j}^{1-\alpha} \mu_{j}^{\alpha} \pi_{j}
$$

Hence,

$$
\operatorname{Tr} C_{\alpha}^{\alpha} \leq n^{\alpha} \operatorname{Tr} Q_{\alpha}^{\alpha}=n^{\alpha} \sum_{j \in J} s_{j}^{1-\alpha} \mu_{j}^{\alpha}
$$

Moreover, since $\alpha<1$,

$$
\frac{1}{\alpha-1} \log \operatorname{Tr} C_{\alpha}^{\alpha} \geq \frac{\alpha}{\alpha-1} \log n+\frac{1}{\alpha-1} \log \left(\sum_{j=1}^{n} s_{j}^{1-\alpha} \mu_{j}^{\alpha}\right)
$$

and hence

$$
\lim _{\alpha \rightarrow 0} D_{\alpha}(\rho \| \sigma) \geq-\log \left(\sum_{j=1}^{n} s_{j}\right)=-\log (\operatorname{Tr} \sigma) .
$$

Moreover, since $\operatorname{supp} \rho=\operatorname{supp} \sigma$, we have

$$
\widetilde{D}_{0}(\rho \| \sigma)=-\log \left(\operatorname{Tr}\left(\Pi_{\rho} \sigma\right)\right)=-\log (\operatorname{Tr} \sigma) .
$$

From (81) and (9) it follows that if $\operatorname{supp} \rho=\operatorname{supp} \sigma$,

$$
\lim _{\alpha \rightarrow 0} D_{\alpha}(\rho \| \sigma) \geq \widetilde{D}_{0}(\rho \| \sigma) .
$$

\subsection{A counterexample to eq.(3) when $\rho$ and $\sigma$ have unequal supports}

Let

$$
\rho=\left(\begin{array}{ll}
1 & 0 \\
0 & 0
\end{array}\right), \quad \sigma=\left(\begin{array}{cc}
1 & c \\
c & 1
\end{array}\right), \quad 0<c<1
$$

Note that $\sigma$ is positive-definite, $\operatorname{supp} \rho \subset \operatorname{supp} \sigma$ and $[\rho, \sigma] \neq 0$. Moreover, $\rho=\Pi_{\rho}$ and hence,

$$
\widetilde{D}_{0}(\rho \| \sigma)=-\log (\operatorname{Tr}(\rho \sigma))=-\log 1=0 .
$$

Let us set $\beta:=\frac{1-\alpha}{2 \alpha}$ with $\alpha \in(0,1)$. Then the eigenvalues of $C_{\alpha}:=\sigma^{\beta} \rho \sigma^{\beta}$ are given by

$$
\lambda_{1}=\frac{1}{2}\left((1+c)^{2 \beta}+(1-c)^{2 \beta}\right) \quad \text { and } \quad \lambda_{2}=0 .
$$

Using the fact that $\lim _{\alpha \rightarrow 0} f(\alpha)^{g(\alpha)}=\exp \left(\lim _{\alpha \rightarrow 0} \frac{\ln f(\alpha)}{(g(\alpha))^{-1}}\right)$, one obtains the following:

$$
\lim _{\alpha \rightarrow 0} D_{\alpha}(\rho \| \sigma)=\lim _{\alpha \rightarrow 0} \frac{1}{\alpha-1} \log \left(\operatorname{Tr} C_{\alpha}^{\alpha}\right)=-\log (1+c)<0 .
$$

From (10) and (11) it follows that in this case,

$$
\lim _{\alpha \rightarrow 0} D_{\alpha}(\rho \| \sigma) \neq \widetilde{D}_{0}(\rho \| \sigma) .
$$




\section{Conclusions}

We prove that the 0-relative Rényi entropy $\widetilde{D}_{0}(\rho \| \sigma)$ is obtainable from the quantum Rényi divergence $\widetilde{D}_{\alpha}(\rho \| \sigma)$ if $\rho$ and $\sigma$ have equal supports. While $\widetilde{D}_{\alpha}(\rho \| \sigma)$ serves as an upper bound for $D_{\alpha}(\rho \| \sigma)$ for arbitrary $\alpha \in[0,1)$ as long as $\operatorname{supp} \rho \subseteq \operatorname{supp} \sigma$, the condition of equal supports is essential for proving that $\widetilde{D}_{0}(\rho \| \sigma)$ also serves as a lower bound for $\lim _{\alpha \rightarrow 0} D_{\alpha}(\rho \| \sigma)$, and hence in establishing equality. This is emphasized by the counterexample given in the last subsection.

Our result implies that for values of the parameter $\alpha$ in the range $[0,1 / 2)$, the relative entropy which plays a fundamental role is the $\alpha$-relative Rényi entropy, and not the quantum Rényi divergence. This claim is further strengthened by the fact that the quantum Rényi divergence does not satisfy the data-processing inequality (a property desirable for a relative entropy) in this parameter range. The latter follows from numerical counterexamples, reported in [5], and also found independently by us. This observation, along with existing results in the literature, suggests that, as far as operationally relevant entropic quantities are concerned, the right parent quantity depends on the value of $\alpha$. For example, there are various operational interpretations of the Rényi entropy of order $\alpha$, for all $\alpha \geq 0$ (see e.g. 20] for a few of these). This is given by $S_{\alpha}(\rho)=-\widetilde{D}_{\alpha}(\rho \| \sigma) \equiv-D_{\alpha}(\rho \| \sigma)$. For a bipartite state, the conditional Rényi entropy of order $\alpha$ arises in entanglement theory (see e.g. [21]). However, this quantity is defined as the difference of two Rényi entropies of order $\alpha$, and so can equivalently be obtained from $D_{\alpha}$ or $\widetilde{D}_{\alpha}$. However, the quantum Chernoff bound, which is an important quatity arising in binary state discrimination, is expressible in terms of the $\alpha$-relative Rényi entropy with $\alpha \in[0,1)$ but not in terms of the quantum Rényi divergence. (See also [2] and [22]). On the other hand, the min- and max- relative entropies, which play pivotal roles in one-shot information theory are obtainable from the quantum Rényi divergence (for $\alpha=1 / 2$ and $\alpha \rightarrow \infty$, respectively) but not from the $\alpha$-relative Rényi entropy. The above observations suggest that it suffices to consider two essential parent quantities from which operationally relevant entropic quantities can be derived. These are the quantum Rényi divergence with parameter $\alpha \geq 1 / 2$, and the $\alpha$-relative Rényi entropy with $\alpha \in[0,1)$.

\section{Acknowledgements}

We are grateful to Yuri Suhov for interesting discussions. We would like to thank Serge Fehr, Marco Tomamichel and Mark Wilde for helpful comments.

\section{References}

[1] M. Ohya and D. Petz, "Quantum Entropy and Its Use," Springer-Verlag, 1993; M. Hayashi, "Quantum Information An Introduction," Springer, 2006;

[2] M. Mosonyi and F. Hiai, "On the quantum Rényi relative entropies and related capacity formulas," IEEE Trans. Inform. Theory, vol. 57, 2474-2487, (2011).

[3] N. Linden, M. Mosonyi and A. Winter, "The structure of Renyi entropic inequalities," arXiv:1212.0248.

[4] M. M. Wilde, A. Winter, D. Yang, "Strong converse for the classical capacity of entanglement-breaking and Hadamard channels," arXiv:1306.1586.

[5] M. Müller-Lennert, F. Dupuis, O. Szehr, S. Fehr and M. Tomamichel, "On quantum Renyi entropies: a new definition and some properties," arXiv:1306.3142. 
[6] R.L. Frank and E.H. Lieb, "Monotonicity of a relative Rényi entropy," arXiv:1306.5358.

[7] S. Beigi, "Quantum Rényi Divergence Satisfies Data Processing Inequality," arXiv:1306.5920.

[8] N. Datta, "Min- and Max-Relative Entropies and a New Entanglement Monotone," IEEE Transactions on Information Theory, vol. 55, no. 6, pp. 2816-2826, June 2009.

[9] F. Dupuis, L. Kraemer, P. Faist, J. M. Renes and R. Renner, "Generalized entropies," arXiv:1211.3141.

[10] R. Renner, "Security of Quantum Key Distribution," arXiv:quant-ph/0512258; Ph.D. thesis.

[11] M. Tomamichel, "A Framework for Non-Asymptotic Quantum Information Theory," PhD Thesis, Department of Physics, ETH Zurich, arXiv:1203.2142.

[12] L. Wang and R. Renner, "One-Shot Classical-Quantum Capacity and Hypothesis Testing," Phys. Rev. Lett. vol. 108, 200501, 2012; M. M. Wilde,"Sequential decoding of a general classical-quantum channel," Proc. Roy. Soc. A, vol. 469, 20130259 (2013)

[13] F. Buscemi and N. Datta, "Entanglement cost in practical scenarios," Phys. Rev. Lett. 106, 130503 (2011).

[14] N. Datta, J. M. Renes, R. Renner and M. M. Wilde, "One-shot lossy quantum data compression," arXiv:1304.2336; to appear in IEEE Trans. Inform. Theory.

[15] F. Brandao, M. Horodecki, N. Ng, J. Oppenheim and S. Wehner, "The second laws of quantum thermodynamics," arXiv:1305.5278.

[16] K.M.R. Audenaert, J. Calsamiglia, R. Munoz-Tapia, E. Bagan, Ll. Masanes, A. Acin and F. Verstraete, "The Quantum Chernoff Bound," Phys. Rev. Lett. 98, 160501 (2007).

[17] H. Araki, "On an inequality of Lieb and Thirring," Lett. Math. Phys., vol. 19, no. 2, pp. 167-170, 1990.

[18] E. H. Lieb and W. Thirring, Studies in mathematical physics, pages 269-297. Princeton University Press, Princeton, 1976.

[19] T. Ogawa and M. Hayashi, "On error exponents in quantum hypothesis testing," IEEE Transactions on Information Theory, vol. 50, no. 6, pp. 1368-1372, June 2004.

[20] G. Adesso, D. Girolami and A. Serafini, Phys. Rev. Lett., vol. 109, 190502 (2012); M. M. Wolf and J. Eisert, New J. Phys. 7, 93 (2005); W. van Dam and P. Hayden, arXiv:quant-ph/0204093.

[21] K. G. H. Vollbrecht and M. M. Wolf, J. Math. Phys. 43, 4299 (2002).

[22] M. Hayashi, Phys. Rev. A 76, 062301, (2007). 